\title{
DOPAMINE REDUCES SLOW OUTWARD CURRENT AND CALCIUM INFLUX IN BURST-FIRING NEURON R15 OF APLYSIA
}

\author{
D. V. LEWIS,${ }^{*} \ddagger^{2}$ G. B. EVANS, $\S^{3}$ AND W. A. WILSON $\S$ \\ Departments of ${ }^{*}$ Medicine, $\ddagger$ Pediatrics, and $§$ Pharmacology, Duke University Medical Center, Durham, North Carolina 27710
}

Received January 24, 1984; Revised June 11, 1984; Accepted June 15, 1984

\begin{abstract}
Dopamine's effect on calcium influx into the bursting neuron, R15, of Aplysia californica was tested by tail current measurements and by measurement of absorbance of intracellular Arsenazo III, a calcium-sensitive indicator. Slow outward tail currents were elicited by subthreshold depolarization in voltage clamp and were demonstrated to be dependent upon transient increases in intracellular calcium activity, $\left((\mathrm{Ca})_{i}\right)$, using calciumfree seawater, calcium hlockers $\left(\mathrm{Mn}^{2+}\right.$ and $\left.\mathrm{La}^{3+}\right)$, and intracellular injection of EGTA. Dopamine reduces these tail currents as it reduces the slow inward current. Next, the transient elevations of $(\mathrm{Ca})_{i}$ accompanying subthreshold depolarization were measured directly in Arsenazo III-loaded neurons. Dopamine did not reduce the rise in $(\mathrm{Ca})_{\mathrm{i}}$ measured in the soma during depolarization. However, when absorbance of the axodendritic region was monitored, dopamine did reduce calcium influx. Voltage monitoring in the axon indicated that the reduced calcium influx could not simply be ascribed to altered space clamp. In keeping with the apparent axodendritic location of dopamine action, isolation of the soma by ligation of the axon markedly reduced the dopamine response. Dopamine seems to reduce calcium influx into R15, but this effect is topographically limited to nonsomatic membrane, an area of the neuron not usually monitored in optical studies of $(\mathrm{Ca})_{i}$.
\end{abstract}

The endogenous bursting rhythm of the neuron R15 of Aplysia californica can be modulated by both natural synaptic input (Wilson and Wachtel, 1978) and artificial application of putative neurotransmitters (Gospe and Wilson, 1980). Specifically, dopamine profoundly inhibits bursting in R15 and markedly alters the current-voltage (I-V) curve of the cell. The slow inward current (SIC) which underlies the depolarizing phase of the bursting cycle (Wilson and Wachtel, 1974) is no longer detectable by voltage clamp techniques after dopamine application. The apparent absence of the SIC is manifest in the I-V curve as a loss of the negative slope resistance region in the subthreshold voltage range (Gospe and Wilson, 1980).

There are several possible explanations for the effect of dopamine on R15. The SIC is thought to be either an inward sodium (Barker and Smith, 1978) or calcium (Gorman et al., 1982) current or perhaps a combined sodium and calcium current. Therefore, dopamine could be reducing calcium and/ or sodium conductance underlying the SIC. Alternatively, it has been suggested that dopamine increases potassium conductance (Ascher, 1972), which then might summate with and obscure the SIC, producing a net outward current during small depolarizations.

We have attempted to test one of these hypotheses; i.e., that dopamine reduces calcium influx into $\mathrm{R} 15$ during small depo-

\footnotetext{
${ }^{1}$ This work was supported by United States Public Health Services Grant NS-18187.

${ }^{2}$ To whom correspondence should be addressed, at Box 3430, Duke University Medical Center, Durham, NC 27710.

${ }^{3}$ Present Address: Beckton Dickenson Research Center, Computer Services Department, P. O. Box 12016, Research Triangle Park, NC 27709 .
}

larizing commands. To do so, we have used two approaches. First, we demonstrated that dopamine eliminates tail currents which seem to be dependent upon intracellular calcium accumulation. Second, we observed the effect of dopamine on calcium influx using the calcium indicator dye Arsenazo III. The results suggest that dopamine reduces calcium influx into the axodendritic tree of R15 during small depolarizing commands.

\section{Materials and Methods}

Aplysia californica weighing 100 to $200 \mathrm{gm}$ were purchased from Marine Specimens Unlimited (Pacific Palisades, CA) and kept in a marine aquarium at $16^{\circ} \mathrm{C}$. The abdominal ganglion was pinned to the bottom of a chamber immersed in artificial seawater of the following composition (in millimolar concentration): $\mathrm{Na}^{+}, 492 ; \mathrm{Cl}^{-}, 664 ; \mathrm{Mg}^{2+}$, 100 ; $\mathrm{SO}_{4}^{-2}, 30 ; \mathrm{K}^{+}, 10$; and $\mathrm{Ca}^{2+}, 10$. The $\mathrm{pH}$ was buffered using $5 \mathrm{mM}$ Tris at 7.8. All solutions were at room temperature of 21 to $23^{\circ} \mathrm{C}$. The high magnesium level $(100 \mathrm{mM})$ facilitated small current analysis by reducing the incidence of interfering large postsynaptic currents and minimizing uncontrolled distal spiking during voltage clamp depolarizations. Previous use of high magnesium seawater has demonstrated no misleading or damaging effects (Gospe and Wilson, 1980; Lewis, 1984). R15 was exposed by removal of the overlying capsule. In experiments where neuropil absorbance measurements were made, the neurons surrounding R15 were removed prior to placing fiberoptic probes. The single-clectrode voltage clamp (Goldner and Wilson, 1975) was used, employing electrodes filled with $1.5 \mathrm{M} \mathrm{KCl}$ having resistances between 1.5 and 2.0 megohms.

Solutions bathing the cell could be easily changed by a gravity-driven flow-through system allowing complete exchange of the $10-\mathrm{ml}$ bath volume in 3 to $5 \mathrm{~min}$. Manganese $\left(\mathrm{MnCl}_{2}\right)$ and lanthanum $\left(\mathrm{LaCl}_{3}\right)$ when used, were simply added to artificial seawater. Zero-calcium -2 mM EGTA seawater was made by substituting $10 \mathrm{mM} \mathrm{MgCl}_{2}$ for 10 $\mathrm{mM} \mathrm{CaCl} 2$ and adding $2 \mathrm{~mm}$ EGTA to artificial seawater. Dopamine was added to artificial seawater along with ascorbic acid (Gospe and Wilson, 1980) to prevent dopamine oxidation. EGTA was pressure 
injected (Picospritzer, General Valve Corp., Hanover, NJ) into cells through a second micropipette containing $250 \mathrm{~mm}$ EDTA adjusted to $\mathrm{pH} 7.3$ with $\mathrm{KOH}$ and containing $10 \mathrm{mM}$ Arsenazo III to visualize the injection solution. The concentration of $\mathrm{K}^{+}$was altered in seawater by adding or deleting the appropriate amount of $\mathrm{KCl}$.

Arsenazo III absorbance measurements were made using a modification of the previously described single-wavelength microspectrophotometer (Lewis and Wilson, 1982). The machine was modified to a dual-wavelength differential instrument using two monochromators and a chopping system which illuminated the cell with the alternate wavelengths with a chopping rate of $300 \mathrm{~Hz}$. Two of three wavelengths, 570,655 , or $700 \mathrm{~nm}$, were utilized in each experiment (Gorman and Thomas, 1978). When studying absorbance changes in the soma, there was abundant dye in the path in the large soma of R15. Consequently, 570-nm light (at the peak of the absorbance spectrum of Arsenazo III) was markedly attenuated, and attempts to use the $655-/ 570-\mathrm{nm}$ pair produced a noisier, less stable signal than did the $655-/ 700-\mathrm{nm}$ pair. Therefore, in soma absorbance measurements, the $655 / 700 \mathrm{~nm}$ pair was used. When measuring axodendritic absorbance changes, there was little dye in the light path and use of the $655-/ 570-\mathrm{nm}$ pair was satisfactory. Gorman and Thomas (1978) have shown that accurate measurements of changes in intracellular calcium activity $\left((\mathrm{Ca})_{\mathrm{i}}\right)$ can be obtained using either wavelength pair. With the small changes in signal $(<5 \%)$ seen in these experiments, the light intensity changes are essentially linearly proportional to the absorbance changes; thus the chart tracings in the figures are calibrated in absorbance. Absorbance calibrations, expressed as percentage of absorbance change, were $A \%$ $=100 \log \left(I_{1} / I_{2}\right)$, with $A \%$ being the percentage of absorbance, $I_{1}$ being the light intensity after the change in absorbance, and $I_{2}$ being the intensity before the change. Arsenazo III was pressure injected into cells as a $100 \mathrm{~mm}$ solution in deionized water (Sigma grade I Arsenazo III). The intracellular concentrations measured at the soma varied from 0.3 to $0.8 \mathrm{~mm}$ and were determined as previously described (Lewis and Wilson, 1982).

Calcium was iontophoresed into R15 using the method of Gorman and Hermann (1979), and stable outward current transients were obtained prior to dopamine application using iontophoresis currents of 100 to $200 \mathrm{nA}$. Iontophoresis current was generated using a 100 megohm resistor in series with the iontophoresis pipettes utilizing a Grass stimulator as the voltage source.

Statistical significance of changes in current or absorbance values was tested using the appropriate $t$ test, and means and standard deviations are given for each measurement.

Axon ligations were attempted in 25 cells and two met the criteria for successful ligation; i.e., spike overshoot to between +40 and +50 $\mathrm{mV}$, spike undershoot below $-45 \mathrm{mV}$, and no increase in slope conductance in the hyperpolarized region of the I-V curve. For ligation a fine silk suture (8-0) was threaded through a piece of theta tubing pulled to a small fire-polished tip. The silk suture formed a loop and this was placed over the soma and snugged down to the proximal axon about 50 to $100 \mu \mathrm{m}$ from the soma. The loop was slowly tightened while the cell was maintained in voltage clamp, using a micrometer attached to the silk suture. This entire device was held in a micromanipulator. To test for completeness of ligation, inhibition of long duration (Adams, et al., 1980) was triggered by stimulation of the branchial nerve. When ligation was complete, the postsynaptic current was no longer visible. In addition, other spontaneous synaptic activity was undetectable and distal uncontrolled spikes were no longer elicited by depolarizing commands.

\section{Results}

Dopamine effects on the membrane currents. Cells were held in voltage clamp between -45 and $-50 \mathrm{mV}$, and prolonged, 5to 10 - sec-duration commands to between -50 and $-25 \mathrm{mV}$ were issued to elicit the SIC (Fig. $1 A$ ). Large commands, usually above $-25 \mathrm{mV}$, elicited rapid, brief inward current deflections (not shown) signifying distant uncontrolled spikes; such distal spiking was avoided whenever possible to prevent activating tail currents secondary to spikes (Lewis, 1984). With commands to above $-40 \mathrm{mV}$ the SIC typically decays significantly during the command, and upon return to holding potential, a slow outward tail current (SOC) develops (Fig. 1A). The SOC was described by Johnston (1980) and, in agreement with his data,

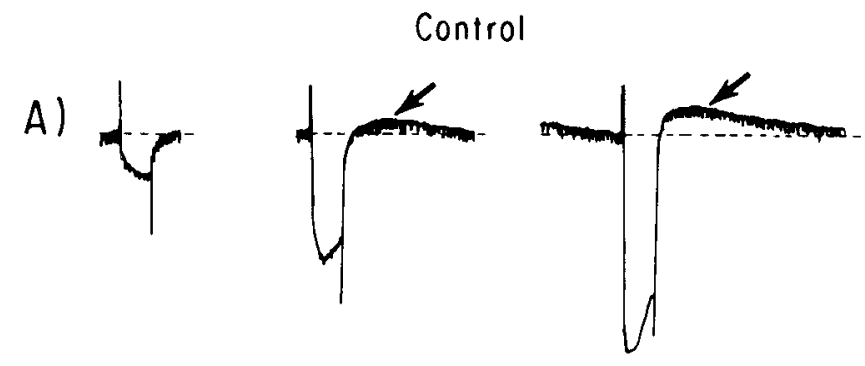

\section{Dopamine}

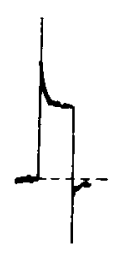

$-40 \mathrm{mV}$

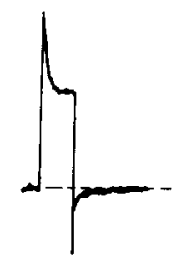

$-35 \mathrm{mV}$

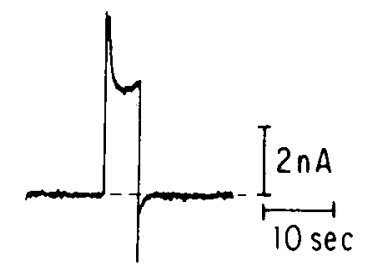

$-30 \mathrm{mV}$

B)
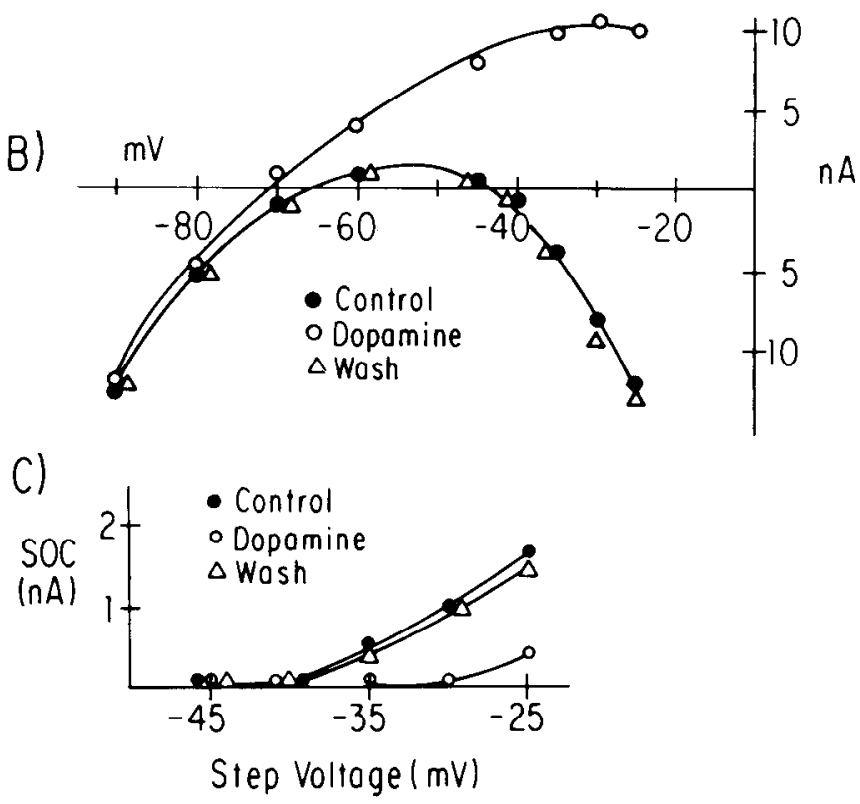

Figure 1. Dopamine effects on the SIC and SOC. A, Top row: Current traces in control cells during commands to $-40,-35$, and $-30 \mathrm{mV}$ from a $-50-\mathrm{mV}$ holding potential. Note SOC (Arrows) after steps. Bottom row: Current traces during the same commands in dopamine $(250 \mu \mathrm{M})$. Note SIC and SOC disappearance. $B$, I-V curve of R15 before ( $)$, during $(O)$, and after $(\triangle)$ dopamine bath application $(250 \mu \mathrm{M})$. Current values are peak inward current during command. $C$, Graph of peak values of SOC versus command level (Step Voltage) before (O), during $(O)$ and after wash $(\Delta)$ of dopamine. All records are from the same cell.

we observed the SOC to peak in 5 to $10 \mathrm{sec}$ and to decay to base line in 20 to $30 \mathrm{sec}$ (at $-50 \mathrm{mV}$ holding).

Bath application of dopamine to R15 eliminates the SIC and negative slope resistance region in the subthreshold voltage range of the I-V curve in a dose-dependent manner (Gospe and Wilson, 1980) (Fig. 1, $A$ and $B$ ). Maximum dopamine effects are seen at 250 to $500 \mu \mathrm{M}$ concentration (Gospe and Wilson, 1980), and these levels were used in our experiments to maximize changes of optical signals which might correlate with dopamine's effect on currents. Dopamine not only eliminates the SIC but reduces the SOC as well (Fig. $1, A$ and $C$ ). In six cells tested, $500 \mu \mathrm{M}$ dopamine reduced the SOC from $2.15 \pm$ $1.0 \mathrm{nA}$ to $0 \pm 0 \mathrm{nA}(p<0.005)$, using moderate depolarization 
to -35 or $-30 \mathrm{mV}$. Recovery after wash was to $1.75 \pm 0.8 \mathrm{nA}$. Dihydroergotamine, which blocks the effect of dopamine on the SIC (Gospe and Wilson, 1981), also blocked its effect on the SOC. In all cells, dopamine produced a 3- to 6-nA outward shift of the holding current at $-50 \mathrm{mV}$, possibly due to reduction of persistent. SIC, which is activated at the holding potential.

Calcium dependance of SOC. Johnston (1980) showed that the SOC was blocked by calcium channel blockers, $\mathrm{Co}^{2+}$ and $\mathrm{Mn}^{2+}$, and suggested that the SOC was a calcium-dependent current. We also tested the sensitivity of the SOC to intracellular calcium accumulation by application of calcium blockers $\mathrm{Mn}^{2+}$ and $\mathrm{La}^{3+}$, by washing cells in zero-calcium $-2 \mathrm{mM}$ EGTA seawater, and by injecting EGTA intracellularly to buffer rises in $(\mathrm{Ca})_{i}$. In these experiments, the SIC was measured as the peak inward current deflection during the command, and the SOC was measured as the peak magnitude of the tail following the command, both relative to the holding current as an arbitrary zero base line.

In five cells washed in $25 \mathrm{mM} \mathrm{Mn}^{2+}$ for $20 \mathrm{~min}$ (Fig. 2A), SOC amplitude was $0.35 \pm 0.13 \mathrm{nA}$ prior to $\mathrm{Mn}^{2+}$ exposure and $0 \pm 0 \mathrm{nA}$ in $\mathrm{Mn}^{2+}(p<0.01)$. SIC amplitude was $2.28 \pm 0.71$ $\mathrm{nA}$ before and $0.43 \pm 0.08 \mathrm{nA}$ in $\mathrm{Mn}^{2+}(p<0.02)$. Lanthanum was applied ( $5 \mathrm{mM})$ to only two cells, eliminating the SOC in both and reducing the SIC to zero in one and to $30 \%$ of control amplitude in the other.

Washing cells in zero-calcium $2 \mathrm{~mm}$ EGTA seawater (Fig. $2 B)$ reduced the $\mathrm{SOC}$ from $1.75 \pm 0.86 \mathrm{nA}$ to $0 \pm 0 \mathrm{nA}(n=6$, $p<0.01)$ and the SIC from $8.3 \pm 3.6$ to $4.1 \pm 1 \mathrm{nA}(p<0.05)$. Both the changes duc to manganese and those due to zero calcium were reversible upon washing the cells in normal seawater.

Ten cells were injected with EGTA; SOC amplitude prior to injection but with the injection pipette in the cell was $1.36 \pm$ $0.56 \mathrm{nA}$ and after injection was $0.14 \pm 0.25 \mathrm{nA}(p<0.01)$ (Fig. $2 C$ ). SIC amplitude prior to injection was $7.66 \pm 3.88 \mathrm{nA}$ and
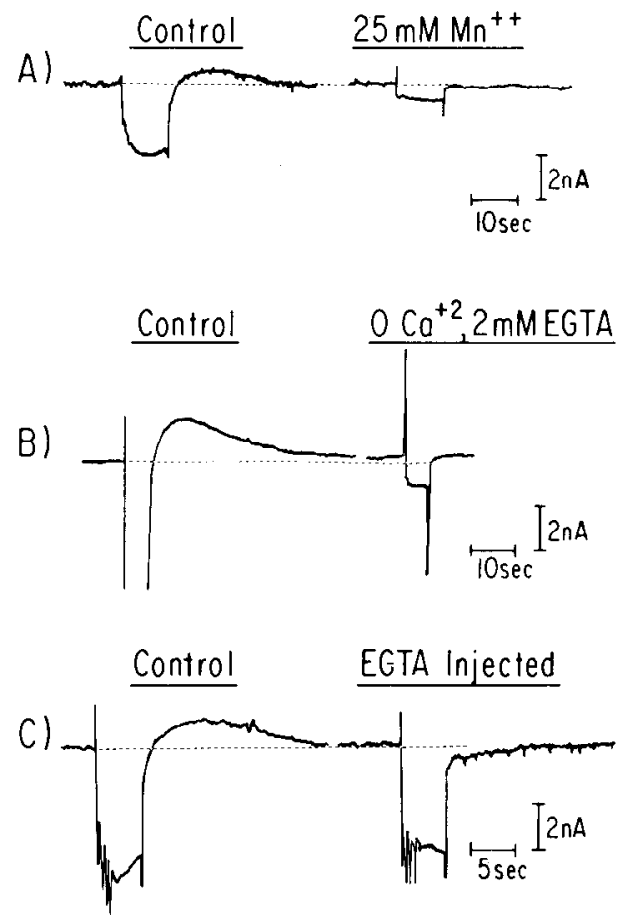

Figure 2. Calcium dependence of SOC. A, Steps from $-46 \mathrm{mV}$ to $-42 \mathrm{mV}$ before and during bath application of $25 \mathrm{mM} \mathrm{Mn}^{++}$. $B$, Steps from -50 to $-35 \mathrm{mV}$ before and during bathing in zero-calcium $-2 \mathrm{mM}$ EGTA seawater. $C$, Steps from -40 to $-30 \mathrm{mV}$ before and after intracellular injection of EGIA. (Peak SIC in Control $(B)$ was 14.5 $\mathrm{nA}$; the trace was truncated to accommodate spacing.) post injection was $5.28 \pm 2.40 \mathrm{nA}(p<0.02)$. EGTA produced a slight ( 1 to $2 \mathrm{nA}$ ) increase in inward holding current in 6 of 10 cells, but no signs of cellular injury (Lewis, 1984) were found.

We conclude from these experiments that the SOC is dependent upon increased intracellular calcium due to calcium influx during the small depolarizing commands. Although the SOC is calcium activated, we could not demonstrate that it was a potassium current. The magnitude of the SOC following a constant depolarizing command decreased as the holding potential was made more hyperpolarized and became undetectable between -70 and $-80 \mathrm{mV}$; however, hyperpolarization as far as $-110 \mathrm{mV}$ did not invert the SOC. Also, cells were bathed in seawater containing $1 \mathrm{mM}, 10 \mathrm{mM}$, and $30 \mathrm{mM} \mathrm{K}^{+}$for 30 to 45 min at each concentration. The altered $\mathrm{K}^{+}$had no significant effect on the amplitude of the SOC following the moderate depolarizations used in these experiments (Fig. 3). The SOCs following commands to between -30 and $-25 \mathrm{mV}$ were measured, and the ratio of SOC amplitude in $1 \mathrm{mM} \mathrm{K} \mathrm{K}^{+}$to amplitude in $10 \mathrm{mM} \mathrm{K}^{+}$was $1.38 \pm 0.54(n=4$ cells $)$. The ratio of SOC amplitude in $30 \mathrm{mM} \mathrm{K} \mathrm{K}^{+}$to amplitude in $10 \mathrm{mM} \mathrm{K}^{+}$was $1.02 \pm$ 0.26 ( $n=5$ cells). Although suggestive of an increase in SOC amplitude in low $\mathrm{K}^{+}$, neither of these ratios were significantly different from $1(p>0.1)$. The spike undershoot became $5.6 \pm$ $1.2 \mathrm{mV}$ more hyperpolarized in $1 \mathrm{mM} \mathrm{K}^{+}$and $13.4 \pm 3.9 \mathrm{mV}$ less hyperpolarized in $30 \mathrm{mM} \mathrm{K} \mathrm{K}^{+}$.

Dopamine and calcium influx into the soma in R15. Because dopamine blocked the calcium-dependent SOC, we hypothesized that it would reduce calcium influx. Therefore, cells were loaded with Arsenazo III and fiberoptic probes were placed on the exposed somata to measure absorbance changes during depolarizing commands. The diameter of the fiberoptics, 250 $\mu \mathrm{m}$, approximated the soma diameter so that only the somatic absorbance changes were recorded. Seven cells were successfully studied, using 10 -sec commands to between -30 and -25 $\mathrm{mV}$ in four cells, to $-20 \mathrm{mV}$ in two cells, and to $-15 \mathrm{mV}$ in one cell. The larger depolarizations often elicited distant uncontrolled spiking; however, this was tolerated because the spikes did not invade the soma and therefore presumably did not affect our somatic absorbance changes. A signal averager was used to sum 10 to 15 absorbance traces in five cells with small absorbance changes, but this was unnecessary in two cells with exceptionally good absorbance signals. Bath application of dopamine $(500 \mu \mathrm{M})$ eliminated the SIC and SOC in all cells (Fig. 4) but had no consistent effect on the absorbance changes. Absorbance increased $0.38 \pm 0.27 \%$ during commands before
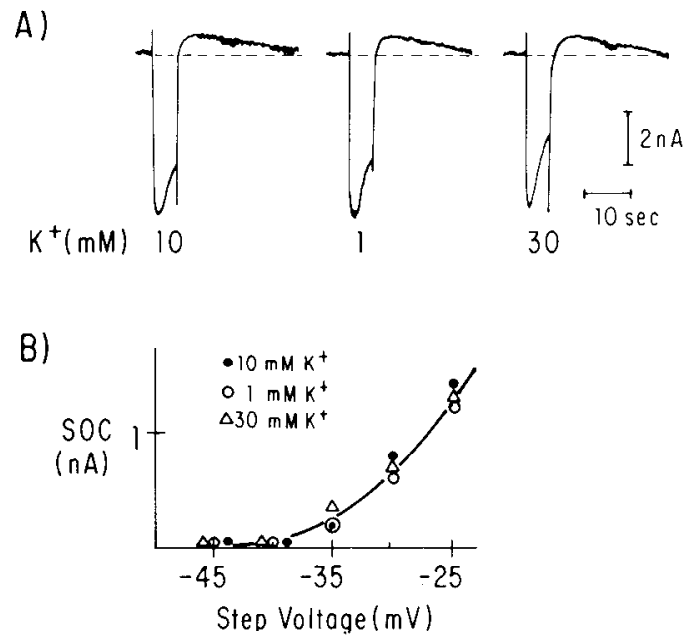

Figure 3. SOC and extracellular potassium. A, Steps from $-50 \mathrm{mV}$ to $-30 \mathrm{mV}$ in 10,1 , and $30 \mathrm{~mm}$ extracellular potassium. $B$, Plot of the peak value of $\mathrm{SOC}$ (holding potential $-50 \mathrm{mV}$ ) versus command level (Step Voltage) in different levels of extracellular $\mathrm{K}^{+}$. 
A)

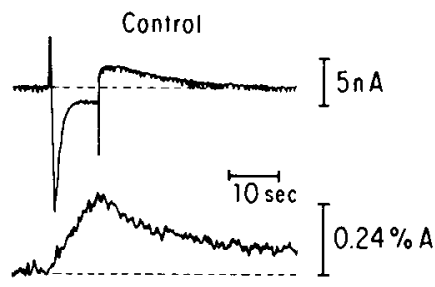

B)
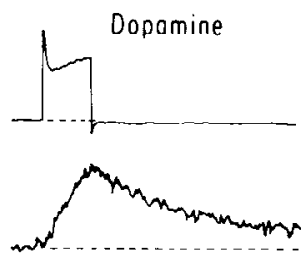

c)

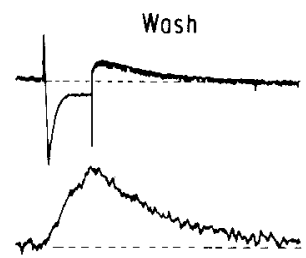

Figure 4. Dopamine and somatic absorbance changes. Current (upper) and absorbance (655-700 $\mathrm{nm}$ ) (lower) traces measured at the soma are shown before $(A)$, during $(B)$, and after $(C)$ wash off of dopamine $(500 \mu \mathrm{M})$. Note no change in the somatic absorbance trace during dopamine treatment although the SIC and SOC are no longer seen. Absorbance traces are not averaged in this cell which has exceptionally good Arsenazo III signal.

dopamine, $0.35 \pm 0.34 \%$ during dopamine application, and 0.34 $\pm 0.27 \%$ after washing off dopamine, showing no statistically significant change.

Somatic calcium influx persisted in dopamine-treated cells, but the SOC was eliminated. This dissociation could indicate that the calcium entering the soma is somehow prevented by dopamine from activating outward current. To test this hypothesis, we iontophoresed calcium into the soma of R15 near the membrane surface and were able to elicit outward currents, presumably the calcium-activated potassium currents as described by others (Gorman and Hermann, 1979) (Fig. 5). In five cells tested, the amplitude of the calcium-activated outward current in control was $7.78 \pm 1.78 \mathrm{nA}, 6.98 \pm 2.6 \mathrm{nA}$ in $500 \mu \mathrm{M}$ dopamine, and 7.5 $\pm 3.8 \mathrm{nA}$ after washing off dopamine. The change was not significant $(p>0.1)$.

Dopamine and calcium influx into the axodendritic region of R15. Ascher (1972) states that dopamine iontophoresed on the soma of R15 produces no current change, whereas iontophoresis into the axodendritic region elicits typical responses; this observation has also been made in our laboratory (unpublished results). Therefore, we attempted to measure the effect of bathapplied dopamine upon calcium influx in the axodendritic region of $\mathrm{R} 15$. The neurons surrounding $\mathrm{R} 15$ were carefully removed and the $250-\mu \mathrm{m}$ fiberoptic probes were placed, one on each side of the mass of neuropil underlying the soma of R15. The soma was filled with Arsenazo III, and as the dye diffused into the cell processes, a small increase in absorbance at the isosbestic point, $570 \mathrm{~nm}$, was clearly observed. The soma was visible above the probes and out of the light path. After each experiment, the fiberoptics were withdrawn and the axon was clearly visible, stained purple by the dye in the mass of tissue which had been between the fiberoptics. We could not see smaller processes, however, since our observations were limited to a dissecting microscope. Nine cells were successfully studied, and very small increases in absorbance were observed during
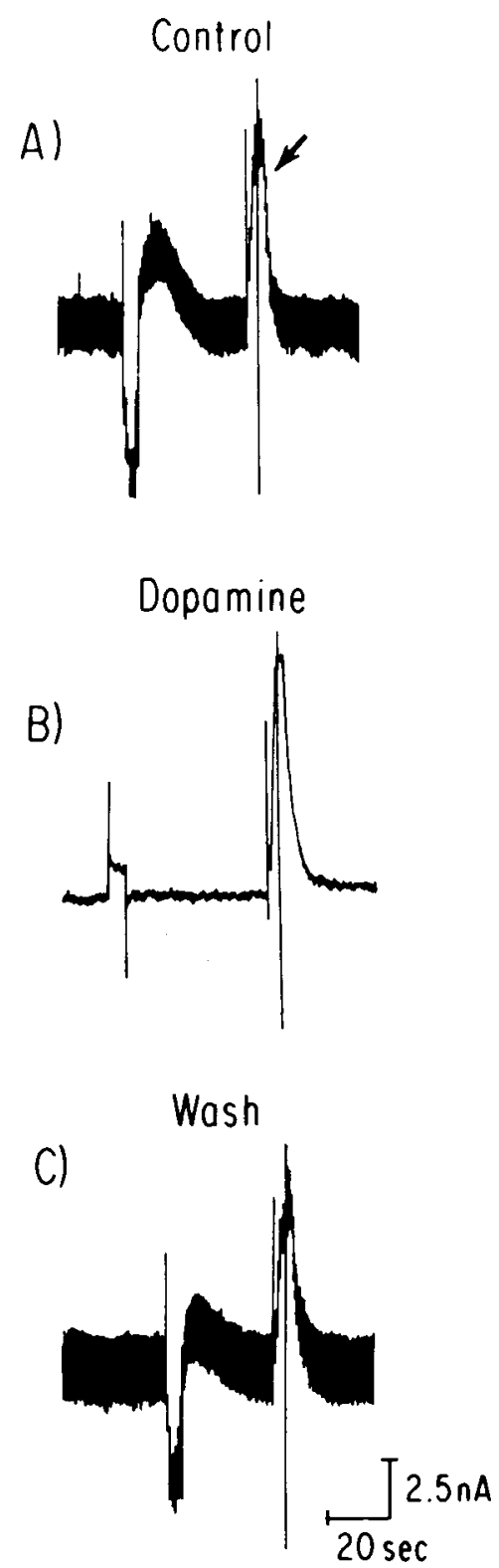

Figure 5. Persistence of somatic calcium-activated outward current during dopamine application. A, Control cell is depolarized from -40 $\mathrm{mV}$ to $-35 \mathrm{mV}$ for $4 \mathrm{sec}$ eliciting the SIC and SOC; then calcium is iontophoresed near the inner surface of the soma membrane producing a transient outward current response (arrow). $B$, Bathing the cell in dopamine $(500 \mu \mathrm{M})$ eliminates the SIC and SOC during the depolarization, but the response to calcium iontophoresis is not reduced. $C$, After washing off dopamine, the SIC and SOC return. The repetitive downward deflections in the current trace in $A$ and $C$ are periodic EPSCs which are usually inhibited by bath-applied dopamine, hence their absence in $B$.

10-sec steps to between -30 and $-25 \mathrm{mV}$ (Fig. 6). The absorbance increase was $0.056 \pm 0.024 \%(n=9)$ prior to dopamine, $0.021 \pm 0.010 \%$ during dopamine $(500 \mu \mathrm{M})$ application, and $0.043 \pm 0.020 \%$ after washing off dopamine. The decrease with dopamine and recovery with wash were both significant $(p<$ 0.01 ). Signal averaging was used in all cells due to the low signal-to-noise ratio with such small absorbance changes.

Nonuniform space clamp as a source of artifact. The apparent decrease of calcium influx in the axodendritic region in dopamine could result from a decrease in the length constant of R15 due to dopamine. Specifically, if dopamine reduced membrane resistance, depolarization of the axon might be less extensive 

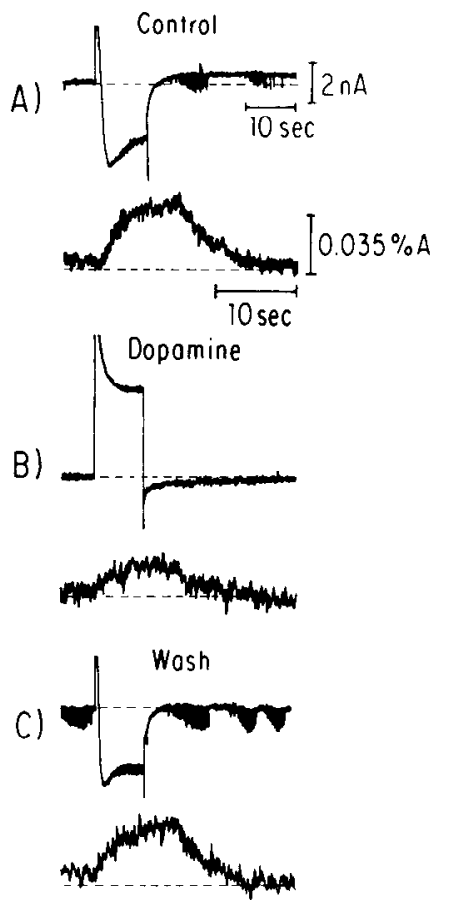

Figure 6. Effect of dopamine on axodendritic absorbance changes. $A$, Current trace (upper) and absorbance trace (lower) $(655-570 \mathrm{~nm}$ ) during a 10 -sec command from -50 to $-25 \mathrm{mV}$. The absorbance traces in $A, B$, and $C$ are on a different time scale; because they were photographed from the display of the averaging computer, each trace is an average of 10 commands. The current traces are one of the unaveraged single commands used to generate the average absorbance change and were traced from a chart recorder graph. $A$, Control; $B$, same cell bathed in $500 \mu \mathrm{M}$ dopamine; $C$, same ccll after washing off dopamine.

in dopamoine and thereby would activate less calcium conductance during the test depolarizations. We tested the effect of dopamine on axon space clamp in two cells. Single-electrode voltage clamp was applied at the soma and the axon was impaled distally, just before it entered the septum dividing the right and left halves of the abdominal ganglion. The axon was visualized by injecting the soma with Arsenazo III. The point of impalement was approximately $300 \mu \mathrm{m}$ from the soma as measured using a calibrated eyepiece in the dissecting microscope. This point was at the distal edge of the optical field used in the absorbance measurement experiments. Axon voltage was well controlled; prior to dopamine application, soma depolarizations resulted in axonal depolarizations which were between 0.5 and $1.5 \mathrm{mV}$ more depolarized than during dopamine application (Fig. $7 A$ ). Thus, in the axon region monitored optically, the depolarizations attained in control and in dopamine were probably within $1 \mathrm{mV}$ of each other.

The maximum change in axonal absorbance expected on the basis of altered space clamp could be estimated if the relationship between level of depolarization and increase in absorbance was known. In six of the cells used for the axonal absorbance recordings, test depolarizations to levels between -37 and 5 $\mathrm{mV}$ were performed in the absence of dopamine. The peak increase in absorbance is plotted against the level of depolarization (Fig. $7 B$ ). The slope of these lines is $0.0045 \pm 0.002 \%$ absorbance change $/ \mathrm{mV}$. Thus, if the entire region optically monitored were $1 \mathrm{mV}$ less depolarized in dopamine, a decrease in absorbance of $0.0045 \%$ might be expected on the basis of space clamp alteration. The mean decrease in peak absorbance was $0.035 \%$ or nearly 8 -fold that expected from space clamp alterations alone, based on these best case estimates.

Isolated somatic currents in R15. If, as the absorbance meas-
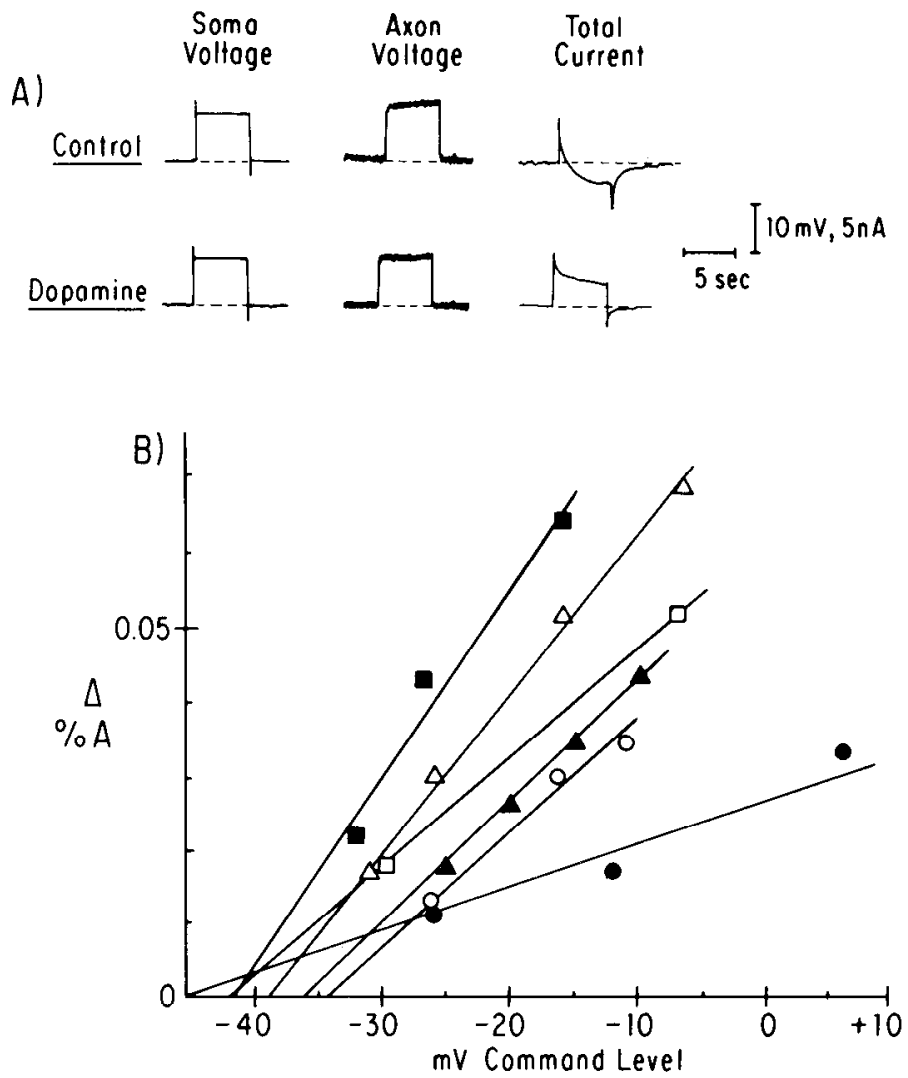

Figure 7. Axon voltage changes and absorbance versus command voltage in axodendritic region. A, Top row (from left to right): Voltage trace at soma during depolarization from -50 to $-40 \mathrm{mV}$, voltage trace from axon $300 \mu \mathrm{m}$ distal from the soma, and current trace at the soma, all before dopamine. Bottom row: repeat step while cell was bathed in $500 \mu \mathrm{M}$ dopamine. $B$, Graph of the absorbance increase (ordinate) versus the command potential (abscissa) for six cells. Lines were drawn by hand and slopes were taken from the lines.

urements indicate, dopamine acts primarily on the axodendritic calcium influx to produce its profound effects on the I-V curve (see Fig. $1 B$ ), this suggests that elimination of the axodendritic portion of the cell should eliminate the dopamine response. To test this hypothesis we attempted to ligate the axon of R15 within 50 to $100 \mu \mathrm{m}$ of the soma. Most commonly, ligation severely damaged the cell, markedly increasing slope conductance and blocking spike generation, but 2 of 25 cells were successfully ligated (see "Materials and Methods" for criteria of successful ligation). Ligation was considered complete when spontaneous and evoked postsynaptic currents were absent. Prior to ligation, stimulation of the branchial nerve elicited a prolonged outward current during which the SIC appeared to be blocked (Fig. 8A). After ligation, the SIC was reduced or eliminated, and stimulation of the connective elicited no detectable response (Fig. $8 A$ ). In addition, the SOC following voltage clamp commands up to $-15 \mathrm{mV}$ was eliminated by ligation in both cells, although slow post-tetanic outward tail currents could be elicited by long bursts of driven spikes.

The I-V curve of one of the ligated cells is shown in Figure $8 B$. This cell showed a normal response to dopamine prior to ligation. After ligation the cell had lost its SIC, but there was no evidence of nonspecific conductance increase. Dopamine had a very small effect on the I-V curve after ligation. This cell did not burst after ligation but would fire long trains of spikes with small depolarizing current injection or fire slowly repetitive spikes without depolarizing current (Fig. $8 \mathrm{C}$ ). The other ligated cell retained some negative slope resistance region after ligation and showed spontaneous bursts of five to seven spikes 
A)

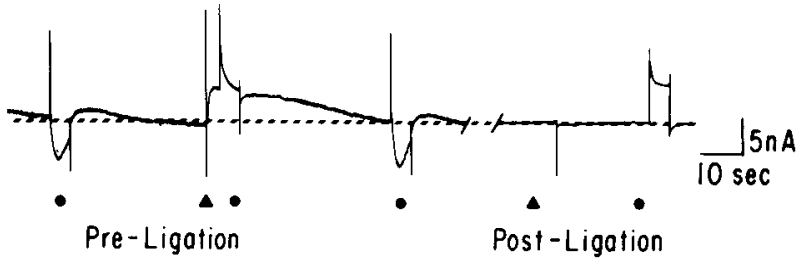

B)

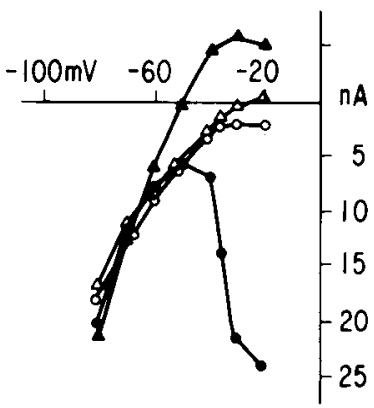

C)

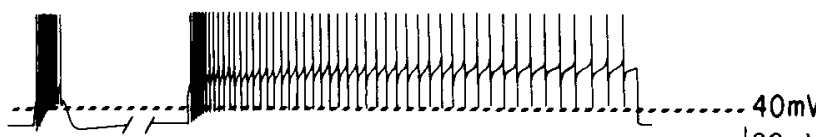

$20 \mathrm{mV}$

Figure 8. Axonal ligation. A, Pre-ligation current record (holding potential $-50 \mathrm{mV}$ ) shows steps to $-35 \mathrm{mV}$ for $5 \mathrm{sec}(-)$ and stimulus to branchial nerve ( $\mathbf{A}$ ) eliciting a prolonged outward current response during which a command does not elicit an inward current. Postligation, the stimulus does not produce an outward current and the same command elicits an outward current with no following SOC. $B$, I-V curves from ligated cell showing dopamine $(500 \mu \mathrm{M})$ effect pre- and post-ligation. $C$, On the left is a typical burst from the cell pre-ligation and on the right is the response to $3 \mathrm{nA}$ injected depolarizing current post-ligation. Note that spike overshoots are clipped by the pen recorder.

separated by hyperpolarized interburst intervals. Dopamine $(500 \mu \mathrm{M})$ did not change the I-V curve nor did it inhibit the bursting activity post-ligation.

\section{Discussion}

These experiments indicate that a significant component of the dopamine effect on R15 may be due to reduced calcium influx. We also must emphasize that our experiments do not exclude additional potential effects of dopamine upon other currents. For instance, a component of the SIC may be sodium mediated (Barker and Smith, 1978; Wilson and Wachtel, 1978) and, therefore, dopamine could reduce sodium influx as well. Ascher (1972) suggests that dopamine increases potassium conductance, and this possibility also is not yet ruled out. Nevertheless, whatever other effects dopamine may have on $\mathrm{R} 15$, it clearly seems to reduce axodendritic calcium influx during small depolarizing commands.

Dopamine also reduces the SOC following subthreshold depolarization of R15. The SOC has been characterized both as a calcium-dependent potassium current (Johnston, 1980) and as a calcium-mediated transient inactivation of persistent SIC (Adams and Levitan, 1981; Kramer and Zucker, 1983). We also found that the $\mathrm{SOC}$ is dependent upon increases in $(\mathrm{Ca})_{\mathrm{i}}$. Our data are more compatible with the SOC being a transient inactivation of persistent SIC because the SOC did not seem to invert with hyperpolarization or to have significant sensitivity to extracellular $\mathrm{K}^{+}$. Regardless of which mechanism accounts for the SOC, its reduction by dopamine is compatible with partial blockade of calcium influx by this neurotransmit- ter. If the $\mathrm{SOC}$ is an axodendritic calcium-dependent potassium current, the decrease in calcium influx seen in Arsenazo III experiments may explain its reduction. If the SOC represents transient inactivation of persistently activated SIC channels, then dopamine could block the SOC by eliminating the SIC. Since the SIC clearly seems to have a component of calcium influx (Gorman, et al., 1982), dopamine would be expected to reduce calcium influx, as was observed.

Calcium influx into the soma of $\mathrm{R} 15$ persisted in dopamine but did not elicit an SOC, even though calcium iontophoresis into the soma elicited a typical calcium-activated potassium current (Gorman and Hermann, 1979). We suggest that the increases in $(\mathrm{Ca})_{\mathrm{i}}$ seen at the soma during these modest depolarizations were insufficient to activate the channels activated by the local iontophoresis of calcium, which probably produces higher local (Ca); levels (Gorman and Hermann, 1979; Gorman and Thomas, 1980). With large depolarizations, occasionally to $-25 \mathrm{mV}$ (Fig. $1 \mathrm{C}$ ) and higher (not illustrated), we observed SOCs in dopamine. The SOCs following greater depolarization may represent calcium-dependent potassium currents activated by larger increases in $(\mathrm{Ca})_{i}$.

Dopamine did reduce axodendritic calcium influx during subthreshold, prolonged depolarizations, and the reduced calcium influx probably accounts for an unknown portion of the outward shift of the shift of I-V curve. The changes in absorbance in the axodendritic region cannot be quantitated in absolute terms of calcium flux because the membrane area and dye concentration within the small processes being monitored are unknown. Nevertheless, the small size of the absorbance changes recorded from the axodendritic region does not necessarily imply that the axon experiences much smaller changes in $(\mathrm{Ca})_{i}$ than does the soma. The small signal reflects primarily the small volume of active tissue in the total region being monitored when the fiberoptics were placed in the neuropil. For example, assuming the axon can be represented as a 30 $\mu \mathrm{m}$-diameter cylinder traversing the $250-\mu \mathrm{m}$-diameter optical field, the profile of the axon would eclipse only $15 \%$ of the face of the receiving fiberoptic. Therefore, the changes in $(\mathrm{Ca})_{\mathrm{i}}$ in the axodendritic region could actually be quite large and could account for a significant portion of the net SIC.

It has been demonstrated that iontophoretic dopamine application is ineffective on the soma but does elicit responses in the axodendritic region (Ascher, 1972). Our finding that dopamine did not reduce calcium influx into the soma, but reduced axodendritic calcium influx, agrees with this topography of the dopamine response. In addition, in two successfully ligated cells, dopamine had only a very small effect on the isolated somata. Although two cells is a small number upon which to base conclusions, we have never encountered an intact R15 neuron in which $500 \mu \mathrm{M}$ dopamine has not produced a dramatic, large change in the I-V curve (Wilson and Wachtel, 1978; Gospe and Wilson, 1980, 1981). These observations suggest that reduction of calcium influx in the axodendritic membrane of R15 might have profound influence on the behavior of these neurons. Dopamine clearly modulates the bursting rhythm of the cell (Gospe and Wilson, 1980). In addition, dopamine eliminates afterpotentials following single spikes in R15 (Lewis, 1984), and the evidence suggests that these afterpotentials are generated in the axodendritic region (Adams and Levitan, 1982; Lewis, 1984) and activated by calcium influx (Lewis, 1984). Therefore, to modulate bursting rhythms powerfully, perhaps calcium influx need be changed only in the axodendritic membrane.

The results of our axon ligation experiments are compatible with the experience of other investigators. Alving (1968) also found ligation a difficult technique, with a success rate of about $10 \%$. One of the successfully ligated cells showed bursting activity, and this bursting was not suppressed by dopamine. 
The other cell did not burst and lost all of its negative slope resistance region. The difference between the two cells probably reflects variation in several factors, e.g., the number of SIC channels on the soma versus the axon, the magnitude of the potassium conductance on the soma versus the axon, the amount of axon remaining proximal to the ligature, and the effects of the unavoidable mechanical injury accompanying ligation. Nevertheless, the results are compatible with the dopamine effect being largely axodendritic. The data of Adams et al. (1980) also agree with our observations. These investigators showed that one effect of stimulating the branchial nerve was elimination of the SIC, as was observed prior to ligation in our cells. Furthermore, dopamine, presumably by eliminating the SIC, attenuated this effect of branchial nerve stimulation as we observed also (results not shown). Thus, dopamine seemed to be acting on the same conductance which was eliminated by inhibition of long duration. We observed that axonal ligation eliminated the synaptic effect of branchial nerve stimulation and markedly reduced the effect of dopamine, suggesting that both phenomena are occurring distally.

One interpretation of the reduced absorbance change during depolarization in dopamine is that dopamine actually reduces calcium conductance. However, this conclusion must remain tentative. Dopamine does alter the space clamp of the axon. Our estimation of the consequences of altered space clamp could be inadequate. We measured the voltage control in the axon only, and Winlow and Kandel (1976) demonstrated numerous smaller dendritic processes arising from the axon in the neuropil under R15. Dopamine could conceivably lead to larger alterations in spacc clamp in these fine processes. If so, dopamine could cause the decreased calcium influx, not by reducing calcium conductance but by making depolarization of these fine processes less uniform. The same considerations apply to the elimination of the SOC. If calcium influx were reduced by poor space clamp, the $\mathrm{SOC}$ would be expected to be sullaller.

In spite of the question of uniform space clamp, certain conclusions can be drawn from these experiments. First, calcium influx during small depolarizations in R15 is not limited to the soma but occurs in the axodendritic region as well. The axodendritic calcium influx seems to account for a significant portion of the SIC and negative slope resistance region based on the axon ligation results. Axonal calcium currents have been seen in other, nonbursting Aplysia neurons by Horn (1968). Therefore, the calcium contribution to the SIC is not limited to the soma, and studies of the SIC must take this extended distribution into account. Second, the SOC may not be generated primarily in the soma. Dopamine eliminated the SOC but did not reduce somatic calcium influx, nor did it reduce outward current responses to injected calcium in the soma. Axonal ligation eliminated the SOC also. These observations suggest that the SOC is generated in the axodendritic region. Finally, these experiments show that absorbance measurements can be made in the axodendritic region of R15. Unlike the soma, this area is rich with presynaptic endings (Frazier, et al., 1967), and other experiments exploring modulation of calcium conductance by synaptic input or by artificially applied transmitters and modulators would seem feasible.

\section{References}

Adams, W. B., and I. B. Levitan (1981) Ionic dependence and charge carriers of the currents underlying bursting in Aplysia neuron R15. Soc. Neurosci. Abstr. 7: 863.

Adams, W. B., and I. B. Levitan (1982) Origin of the depolarizing afterpotential in Aplysia cell R15. Soc. Neurosci. Abstr. 8: 126.

Adams, W. B., I. Parnas, and I. B. Levitan (1980) Mechanism of long lasting inhibition in Aplysia neuron R15. J. Neurophysiol. 44: 11481160 .

Alving, B. O. (1968) Spontaneous activity in isolated somata of Aplysia pacemaker neurons. J. Gen. Physiol. 51: 29-45.

Ascher, P. (1972) Inhibitory and excitatory effects of dopamine on Aplysia neurons. J. Physiol. (Lond.) 225: 173-209.

Barker, J. L., and T. G. Smith, Jr. (1978) Electrophysiological studies of molluscan neurons generating bursting pacemaker potential activity. In Abnormal Neuronal Discharges, N. Chalazonitis and M. Boisson, eds., pp. 359-387, Raven Press, New York.

Frazier, W. T., E. R. Kandel, I. Kupfermann, R. Waziri, and R. E Coggeshall (1967) Morphological and functional properties of identified neurons in the abdominal ganglion of Aplysia californica. J. Neurophysiol. 30: 1288-1351.

Goldner, M. M., and W. A. Wilson (1975) Voltage clamping with a single microelectrode. J. Neurobiol, 6: 411-422.

Gorman, A. L. F., and A. Hermann (1979) Internal effects of divalent cations on potassium permeability in molluscan neurons. J. Physiol. (Lond.) 296: 393-410

Gorman, A. L. F., and M. V. Thomas (1978) Changes in the intracellular concentration of free calcium ions in a pace-maker neurone measured with the metallochromic indicator dye Arsenazo III. J. Physiol. (Lond.) 275: 357-376.

Gorman, A. L. F., and M. V. Thomas (1980) Potassium conductance and internal calcium accumulation in a molluscan neurone. J. Physiol. (Lond.) 308: 287-313.

Gorman, A. L. F., A. Hermann, and M. V. Thomas (1982) Ionic requirements for membrane oscillations and their dependence on the calcium concentration in a molluscan pace-maker neurone. J. Physiol. (Lond.) 327: 185-217.

Gospe, S. M., Jr., and W. A. Wilson, Jr. (1980) Dopamine inhibits burst-firing of neurosecretory cell R15 in Aplysia californica: Establishment of a dose response relationship. J. Pharmacol. Exp. Ther. 214: 112-118.

Gospe, S. M., Jr., and W. A. Wilson, Jr. (1981) Pharmacological studies of a novel dopamine-sensitive receptor mediating burst-firing inhibition of neurosecretory cell R15 in Aplysia californica. .I. Pharmacol. Exp. Ther. 216: 368-377.

Horn, R. (1968) Propagating calcium spikes in an axon of Aplysia. J. Physiol. (Lond.) 281: 513-534.

Johnston, D. (1980) Voltage, temperature and ionic dependence of the slow outward current in Aplysia burst-firing neurones. J. Physiol. (Lond.) 289: 145-157.

Kramer, R. H., and R. S. Zucker (1983) Inactivation of persistent inward current mediates post-burst hyperpolarization in Aplysia bursting pacemaker cells. Soc. Neurosci. Abstr. 9: 510.

Lewis, D. V. (1984) Spike aftercurrents in R15 of Aplysia: Their relationship to slow inward current and calcium influx. J. Neurophysiol. 51: 403-419.

Lewis, D. V., and W. A. Wilson (1982) Calcium influx and poststimulus current during early adaptation in Aplysia giant neurons. J. Neurophysiol. 48: 202-216.

Wilson, W. A., and H. Wachtel (1974) Negative resistance characteristic essential for the maintenance of slow oscillations in bursting neurons. Science 186: 932-934.

Wilson, W. A., and H. Wachtel (1978) Prolonged inhibition in burst firing neurons: Synaptic inactivation of the slow regenerative inward current. Science 202: 772-775.

Winlow, W., and E. R. Kandel (1976) The morphology of identified neurons in the abdominal ganglion of Aplysia californica. Brain Res. 112: 221-249. 